\title{
Novel mutations in the RS1 gene in Japanese patients with X-linked congenital retinoschisis
}

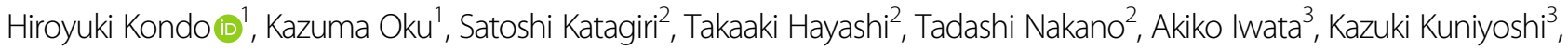 \\ Shunji Kusaka ${ }^{3}$, Atsushi Hiyoshi ${ }^{4}$, Eiichi Uchio ${ }^{4}$, Mineo Kondo ${ }^{5}$, Noriko Oishi', Shuhei Kameya ${ }^{6}$, Atsushi Mizota ${ }^{7}$, \\ Nobuhisa Naoi ${ }^{8}$, Shinji Ueno ${ }^{9}$, Hiroko Terasaki ${ }^{9}$, Takeshi Morimoto ${ }^{10}$, Masayoshi Iwaki ${ }^{11}$, Kazutoshi Yoshitake ${ }^{12}$, \\ Daisuke lejima $^{12}$, Kaoru Fujinami ${ }^{13}$, Kazushige Tsunoda ${ }^{13}$, Kei Shinoda ${ }^{14}$ and Takeshi Iwata ${ }^{12}$
}

\begin{abstract}
X-linked congenital retinoschisis (XLRS) is an inherited retinal disorder characterized by reduced central vision and schisis of the macula and peripheral retina. XLRS is caused by mutations in the RS1 gene. We have identified 37 different mutations in the RS1 gene, including 12 novel mutations, in 67 Japanese patients from 56 XLRS families. We present clinical features of these patients in relation to the associated mutations.
\end{abstract}

$\mathrm{X}$-linked congenital retinoschisis (XLRS) is an inherited retinal disorder that affects central vision and manifests in early childhood ${ }^{1}$. XLRS is the most common inherited retinal disorder; its highest reported prevalence is 14 per 10,000 individuals in Finland ${ }^{1}$. XLRS is characterized by foveal retinoschisis, which occurs in nearly $100 \%$ of patients, whereas peripheral schisis is present in $50 \%$ of XLRS patients ${ }^{1}$. Neuronal dysfunction, manifested as a reduction in the b-wave/a-wave ratio of dark-adapted electroretinograms (DA-ERGs), is also a characteristic of XLRS, although the detection rate for this sign has varied $^{2,3}$. Clinical diagnosis is not easily determined in certain cases because of the wide range of phenotypes, which may include macular and retinal degeneration and secondary complications such as vitreous hemorrhage and retinal detachment; thus, genetic diagnosis is helpful.

XLRS is caused by mutations in the RS1 gene ${ }^{4}$, which encodes retinoschisin, a $24-\mathrm{kDa}$ retina-specific protein

\footnotetext{
Correspondence: Hiroyuki Kondo (kondohi@med.uoeh-u.ac.jp)

${ }^{1}$ Department of Ophthalmology, University of Occupational and Environmental Health, Kitakyushu, Japan

2Department of Ophthalmology, The Jikei University School of Medicine, Tokyo, Japan

Full list of author information is available at the end of the article.
}

secreted by photoreceptors and bipolar cells. Retinoschisin functions as a cell adhesion protein that maintains the synaptic structure of the retina ${ }^{5}$. To date, according to the Human Gene Mutation Database (HGMD; 2018.2 version, https://portal.biobase-international.com), 251 different mutations in this gene are known to cause XLRS. Because of variations in phenotypes among and within families, the genotype-phenotype relationship has not yet been definitively established ${ }^{6,7}$.

We conducted a multicenter observational study at 12 institutions located throughout Japan; this study was approved by the ethics committee of each institution. Signed written informed consent was obtained from all participants or their parents.

Sixty-seven Japanese patients from 56 families with XLRS were studied (Table 1). All patients were male, and their average age was 19.1 years (range: 2 months to 57 years). XLRS was diagnosed based on retinal findings, including the presence of foveal schisis with or without peripheral schisis and a reduced b-wave/a-wave ratio on dark-adapted ERGs as well as family history ${ }^{1}$. The 56 patients included 14 patients with familial XLRS and 42 patients with sporadic XLRS. Medical records were reviewed for all patients who had been identified as carriers of mutations in the RS1 gene. 


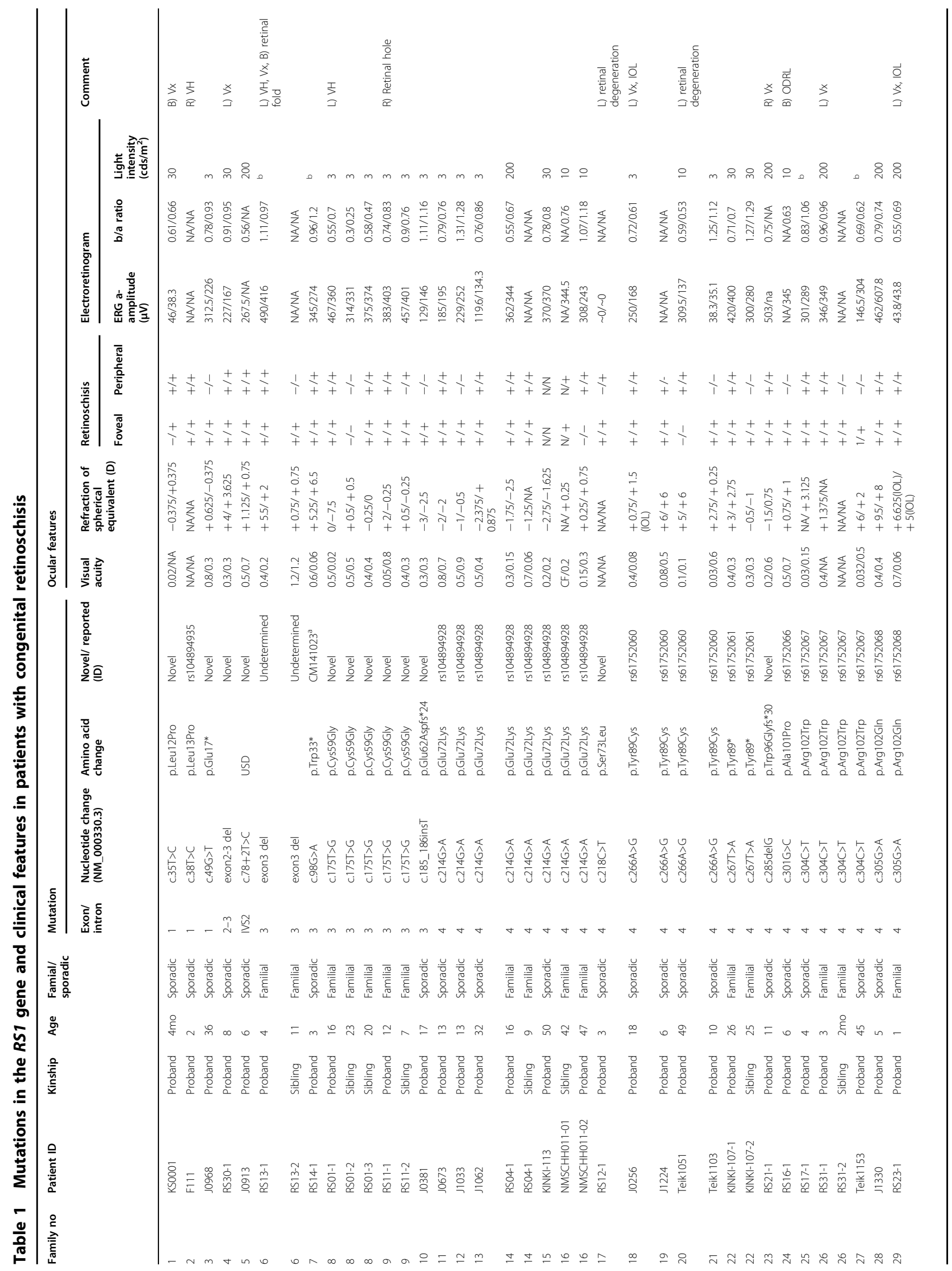




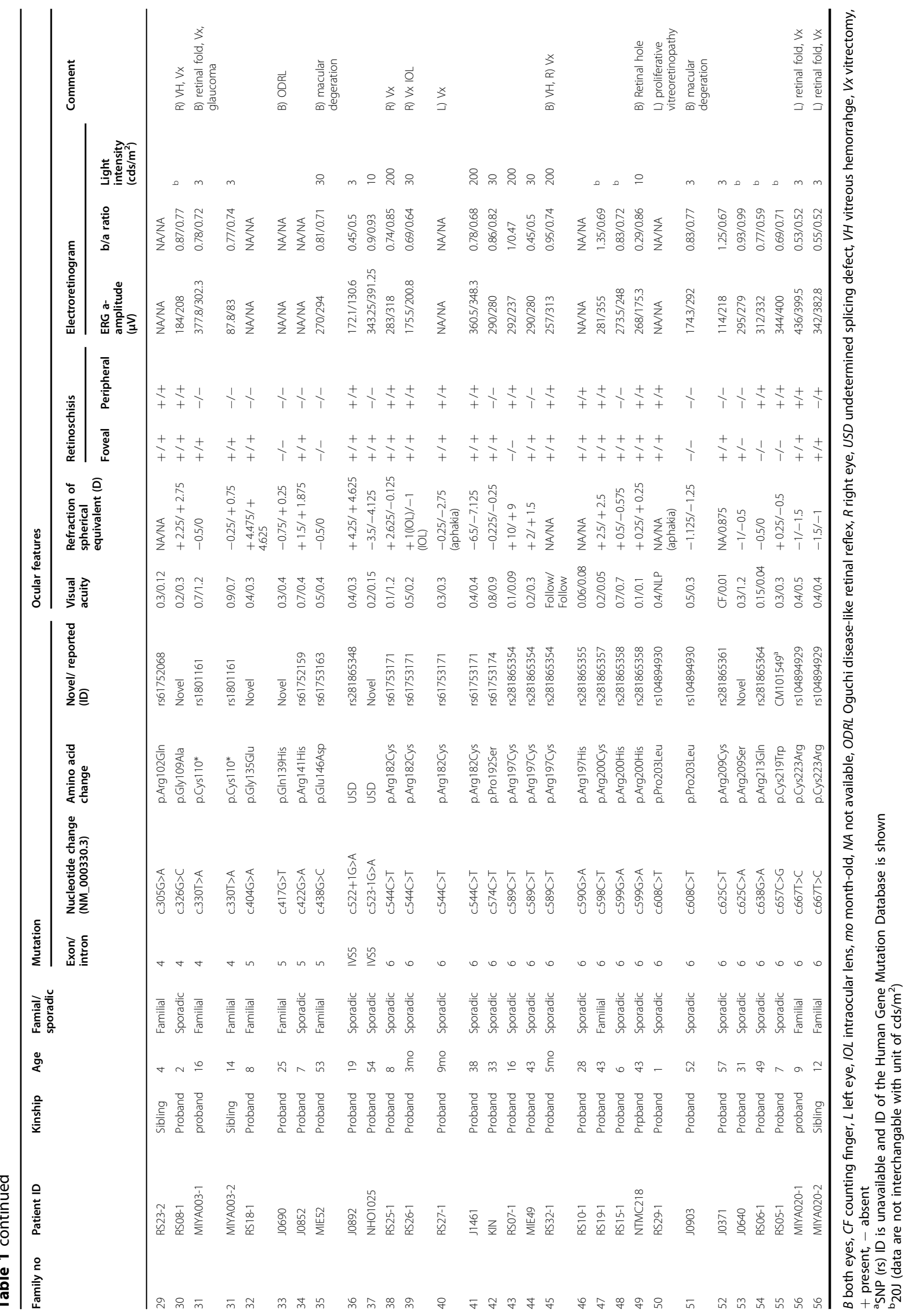




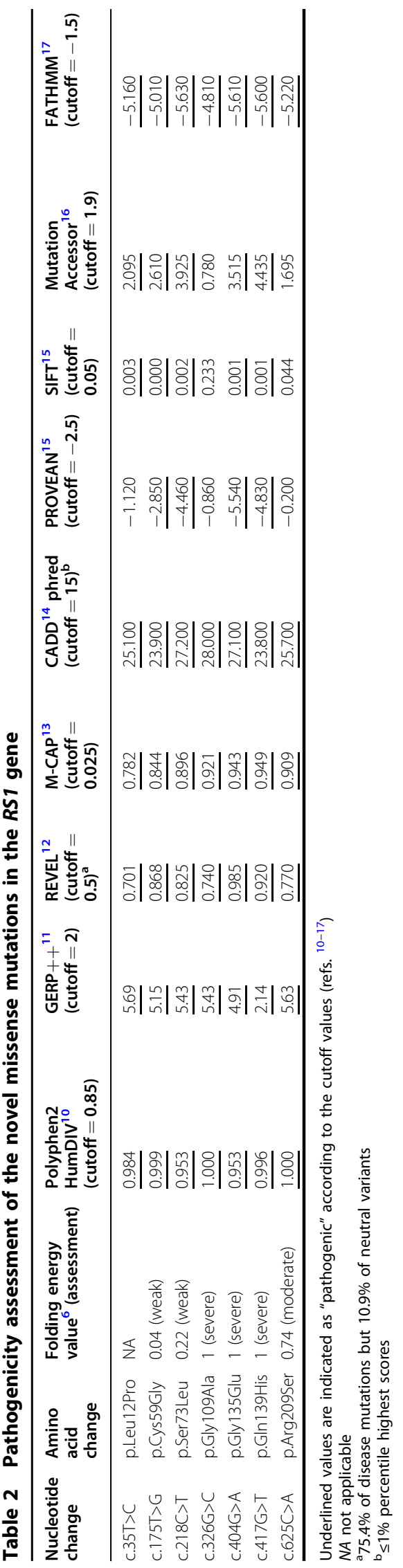

Genomic DNA was extracted from peripheral blood using DNA extraction kits or manual extraction with ethanol. Polymerase chain reaction (PCR) followed by Sanger sequencing was performed on 56 samples for six coding exons of the RS1 gene unless whole-exon deletions were detected via PCR. In brief, oligonucleotide primers for the flanking intron/untranslated region sequences were designed, and PCR was performed, followed by unior bidirectional sequencing depending on the quality of the PCR products. The primer sequences and annealing temperature for PCR for each exon are available on request. The other 11 samples were screened by wholeexome sequencing with at least $30 \times$ coverage for all exons. To identify sequence variations, reference sequences of RS1 (NM_000330.3) were used; variations were numbered based on the cDNA sequence, with +1 corresponding to the first nucleotide of the initiation codon (ATG).

Thirty-seven different mutations in the RS1 gene were identified in the 56 families, including 26 missense, 4 nonsense, 3 splicing, 1 deletion, 1 insertion, and 2 wholeexon deletion mutations (Table 1). Eleven point mutations were novel mutations, and 24 point mutations had previously been reported, based on the HGMD and one recent report (Table 1$)^{8}$. A whole-exon deletion of exon 3 had been reported ${ }^{9}$, whereas a deletion of exons 2 and 3 has not been reported. In our study, DNA break points were not determined, and it is unknown whether the exon 3 deletion that we observed was identical to the known exon 3 deletion at the DNA level.

The frequency of the 11 novel point mutations was assessed using public domain databases. None of these variants were found in human genome variation databases for the Japanese population (the Human Genetic Variation Database (HGVD), http://www.hgvd.genome.med. kyoto-u.ac.jp/) or other population databases, such as the 1000 Genomes Project database (http://www. internationalgenome.org/1000-genomes-browers), the Exome Aggregation Consortium (ExAC) database (http:// www.exac.broadinstitute.org), and the 6500-exome database of the NHLBI-ESP project (ESP6500, http://evs.gs. washington.edu/EVS/). The pathogenicity of the seven novel missense mutations was predicted in silico by nine programs and via folding energy assessments ${ }^{6,10-17}$. Overall, all variants were considered to be pathogenic (Table 2).

Seven of the known mutations were detected in more than one family; in particular, p.Glu72Lys, p.Tyr89Cys, p. Arg182Cys, p.Arg102Trp, p.Arg197Cys, p.Arg200His, and p.Pro203Leu were observed in $6,4,4,3,3,2$, and 2 families, respectively. These mutations have previously been reported in the same population and in other populations $s^{4,7,9}$. Mutation hot spots were suggested instead of founder effects as an explanation of these mutations. 
Overall, the clinical findings of this study were consistent with those of earlier reports, although detailed phenotype-genotype relationships remain undetermined $^{1,3,6,7}$.

Of the 109 phakic eyes for which refractive error (in spherical equivalents) was measured, there were 60 (55.0\%), 5 (4.6\%), and 44 (40.4\%) hypermetropic, emmetropic, and myopic eyes, respectively (Table 1 ). For the hypermetropic eyes, the refractive error ranged from 0.25 to 10.0 diopters $(\mathrm{D})$, and the average error was $+2.7 \mathrm{D}$. For the myopic eyes, the refractive error ranged from -0.125 to $-7.5 \mathrm{D}$, and the average error was $-1.6 \mathrm{D}$. The average difference in refractive error between the two eyes was $1.0 \mathrm{D}$ for 51 patients.

For 125 eyes, the decimal best-corrected visual acuity varied from counting fingers to 1.2 , with a median of 0.3 . For 131 eyes for which retinal status was determined, retinoschisis was present in the macula in 110 eyes $(84.0 \%)$ and in the periphery in 88 eyes $(61.8 \%)$.

DA-ERGs were recorded in 104 eyes using different stimulus intensities; intensities that tended to be higher than those recommended in the standard protocol from the International Society of Clinical Electrophysiology of Vision were used for certain patients ${ }^{18}$. Negative ERGs were more frequently observed in this study $(84.6 \%$, Table 1) than in earlier studies ${ }^{3}$, likely due to the use of higher-intensity light stimuli ${ }^{19}$.

The observed retinal complications included a need for pars plana vitrectomy $(N=16)$; macular or retinal degeneration, including Oguchi disease-like retinal surface abnormalities $(N=6)$; vitreous hemorrhage $(N=5)$; retinal folds $(N=4)$; and congenital glaucoma $(N=1)$.

We sought to establish a possible phenotype-genotype relationship for eyes with truncation mutations (i.e., nonsense, splicing, deletion, insertion, or exon deletion mutations) as opposed to missense mutations. The newly identified mutations do not appear to produce distinct clinical phenotypes compared with reported mutations. However, patients with novel missense mutations did present at an earlier age than those with reported missense mutations (data not shown).

Foveal schisis was more frequently found in eyes with truncation mutations than in those with missense mutations $(100 \%$ versus $78 \%, P=0.0035$, Supplemental Table 1). It is possible that nearly normal foveal structure can only be seen in eyes with missense mutations ${ }^{20}$. Peripheral schisis was found in $50 \%$ and $67 \%$ of eyes with truncation and missense mutations, respectively $(P=$ 0.107).

Compared with eyes with missense mutations, eyes with truncation mutations showed larger b-waves $(P=0.023)$ and higher $\mathrm{b} / \mathrm{a}$ ratios $(P=0.019)$ on DA-ERG, whereas no significant difference was observed for the mean a-wave amplitude (Supplemental Table 2). Differences in patient age, visual acuity, refractive error, and light stimulus settings for DA-ERGs were not significant.

Vincent et al. ${ }^{20}$ reported that truncation mutations were associated with poor visual acuity and a higher probability of a b/a ratio $<1.0$. Our data yielded contradictory results, with higher b-wave amplitude and a greater b/a ratio in eyes with truncation mutations than in eyes with missense mutations. One possible reason for this discrepancy is that the patients with truncation mutations presented at a younger age, which tends to be associated with better preservation of ERG findings ${ }^{21}$. Nonetheless, our study implies that it will be difficult to determine a phenotype-genotype relationship using ERGs.

This study has limitations. Because of the retrospective nature of this investigation, in which only mutationproven cases were selected, the identification rate of the RS1 gene in XLRS has not been determined. A history of clinical findings, including vitreous hemorrhages, may have been missed in certain cases due to only reviewing medical records.

In summary, this study was the largest survey of patients with mutations in the $R S 1$ gene in the Japanese population. The progress of gene therapy for XLRS has reached the clinical trial stage, and exact genetic determinations for each patient could lead to more efficient future treatments ${ }^{22}$.

\section{HGV database}

The relevant data from this Data Report are hosted at the Human Genome Variation Database at https://doi.org/10.6084/m9.figshare.hgv.2408

https://doi.org/10.6084/m9.figshare.hgv.2411

https://doi.org/10.6084/m9.figshare.hgv.2414

https://doi.org/10.6084/m9.figshare.hgv.2417 https://doi.org/10.6084/m9.figshare.hgv.2420

https://doi.org/10.6084/m9.figshare.hgv.2423 https://doi.org/10.6084/m9.figshare.hgv.2426 https://doi.org/10.6084/m9.figshare.hgv.2429 https://doi.org/10.6084/m9.figshare.hgv.2432 https://doi.org/10.6084/m9.figshare.hgv.2435 https://doi.org/10.6084/m9.figshare.hgv.2438 https://doi.org/10.6084/m9.figshare.hgv.2441 https://doi.org/10.6084/m9.figshare.hgv.2444 https://doi.org/10.6084/m9.figshare.hgv.2447 https://doi.org/10.6084/m9.figshare.hgv.2450 https://doi.org/10.6084/m9.figshare.hgv.2453 https://doi.org/10.6084/m9.figshare.hgv.2456 https://doi.org/10.6084/m9.figshare.hgv.2459 https://doi.org/10.6084/m9.figshare.hgv.2462 https://doi.org/10.6084/m9.figshare.hgv.2465 https://doi.org/10.6084/m9.figshare.hgv.2468 https://doi.org/10.6084/m9.figshare.hgv.2471 https://doi.org/10.6084/m9.figshare.hgv.2474 https://doi.org/10.6084/m9.figshare.hgv.2477 https://doi.org/10.6084/m9.figshare.hgv.2480 https://doi.org/10.6084/m9.figshare.hgv.2483 https://doi.org/10.6084/m9.figshare.hgv.2486 https://doi.org/10.6084/m9.figshare.hgv.2489 https://doi.org/10.6084/m9.figshare.hgv.2492 https://doi.org/10.6084/m9.figshare.hgv.2495 https://doi.org/10.6084/m9.figshare.hgv.2498 https://doi.org/10.6084/m9.figshare.hgv.2501 https://doi.org/10.6084/m9.figshare.hgv.2504 https://doi.org/10.6084/m9.figshare.hgv.2507 
https://doi.org/10.6084/m9.figshare.hgv.2510 https://doi.org/10.6084/m9.figshare.hgv.2513

\section{Acknowledgements}

The study was supported by a Japanese Grant-in-Aid for Scientific Research (17K11441 to H.K.) and by the Practical Research Project for Rare/Intractable Diseases (17ek0109282h0001 to T.I.), a program of the Japan Agency for Medical Research and Development.

\section{Author details}

${ }^{1}$ Department of Ophthalmology, University of Occupational and Environmental Health, Kitakyushu, Japan. ${ }^{2}$ Department of Ophthalmology, The Jikei University School of Medicine, Tokyo, Japan. ${ }^{3}$ Department of Ophthalmology, Kindai University Faculty of Medicine, Osakasayama, Japan. ${ }^{4}$ Department of Ophthalmology, Fukuoka University, Fukuoka, Japan. ${ }^{5}$ Department of Ophthalmology, Mie University Graduate School of Medicine, Tsu, Japan. ${ }^{6}$ Department of Ophthalmology, Nippon Medical School Chiba Hokusoh Hospital, Chiba, Japan. ${ }^{7}$ Department of Ophthalmology, Teikyo University, Tokyo, Japan. ${ }^{8}$ Department of Ophthalmology, Miyazaki University, Miyazaki, Japan. ${ }^{9}$ Department of Ophthalmology, Nogoya University Graduate School of Medicine, Nagoya, Japan. ${ }^{10}$ Department of Applied Visual Science, Osaka University Graduate School of Medicine, Osaka, Japan. ${ }^{11}$ Department of Ophthalmology, Aichi Medical University, Nagakute, Japan. ${ }^{12}$ Division of Molecular and Cellular Biology, National Institute of Sensory Organs, National Tokyo Medical Center, Tokyo, Japan. ${ }^{13}$ Division of Vision Research, National Institute of Sensory Organs, National Tokyo Medical Center, Tokyo, Japan. ${ }^{14}$ Department of Ophthalmology, Saitama Medical University, Moroyama, Japan

\section{Conflict of interest}

The authors declare that they have no conflict of interest.

\section{Publisher's note}

Springer Nature remains neutral with regard to jurisdictional claims in published maps and institutional affiliations.

Supplementary information is available for this paper at https://doi.org/ 10.1038/s41439-018-0034-6.

Received: 13 August 2018 Revised: 12 November 2018 Accepted: 9 December 2018.

Published online: 8 January 2019

\section{References}

1. George, N. D., Yates, J. R. \& Moore, A. T. X linked retinoschisis. Br. J. Ophthalmol. 79, 697-702 (1995).

2. Khan, N. W., Jamison, J. A., Kemp, J. A. \& Sieving, P. A. Analysis of photoreceptor function and inner retinal activity in juvenile X-linked retinoschisis. Vision. Res. 41, 3931-3942 (2001)
3. Renner, A. B. et al. ERG variability in X-linked congenital retinoschisis patients with mutations in the RS1 gene and the diagnostic importance of fundus autofluorescence and OCT. Doc. Ophthalmol. 116, 97-109 (2008).

4. Sauer, C. G. et al. Positional cloning of the gene associated with X-linked juvenile retinoschisis. Nat. Genet. 17, 164-170 (1997).

5. Wu, W. W. \& Molday, R. S. Defective discoidin domain structure, subunit assembly, and endoplasmic reticulum processing of retinoschisin are primary mechanisms responsible for X-linked retinoschisis. J. Biol. Chem. 278, 28139-28146 (2003).

6. Sergeev, Y. V. et al. Molecular modeling of retinoschisin with functional analysis of pathogenic mutations from human $X$-linked retinoschisis. Hum. Mol. Genet. 19, 1302-1313 (2010).

7. Shinoda, K., Ishida, S., Oguchi, Y. \& Mashima, Y. Clinical characteristics of 14 japanese patients with X-linked juvenile retinoschisis associated with XLRS1 mutation. Ophthalmic Genet. 21, 171-180 (2000).

8. Katagiri, S. et al. Clinical features of a toddler with bilateral bullous retinoschisis with a novel RS1 mutation. Am. J. Ophthalmol. Case Rep. 5, 76-80 (2017).

9. Functional implications of the spectrum of mutations found in 234 cases with X-linked juvenile retinoschisis. The Retinoschisis Consortium. Hum. Mol. Genet 7. 1185-1192 (1998)

10. Adzhubei, I. A. et al. A method and server for predicting damaging missense mutations. Nat. Methods 7, 248-249 (2010).

11. Davydov, E. V. et al. Identifying a high fraction of the human genome to be under selective constraint using GERP++. PLoS Comput. Biol. 6, e1001025 (2010).

12. Ioannidis, N. M. et al. REVEL: an ensemble method for predicting the pathogenicity of rare missense variants. Am J Hum Genet 99, (877-885 (2016).

13. Jagadeesh, K. A. et al. M-CAP eliminates a majority of variants of uncertain significance in clinical exomes at high sensitivity. Nat. Genet. 48, 1581-1586 (2016).

14. Kircher, M. et al. A general framework for estimating the relative pathogenicity of human genetic variants. Nat. Genet. 46, 310-315 (2014).

15. Kumar, P., Henikoff, S. \& Ng, P. C. Predicting the effects of coding nonsynonymous variants on protein function using the SIFT algorithm. Nat. Protoc. 4, 1073-1081 (2009).

16. Reva, B., Antipin, Y. \& Sander, C. Predicting the functional impact of protein mutations: application to cancer genomics. Nucleic Acids Res. 39, e118 (2011).

17. Shihab, H. A. et al. Predicting the functional, molecular, and phenotypic consequences of amino acid substitutions using hidden Markov models. Hum. Mutat. 34, 57-65 (2013)

18. Robson, A. G. et al. ISCEV guide to visual electrodiagnostic procedures. Doc Ophthalmol. 136, 1-26 (2018).

19. Marmor, M. F. et al. ISCEV Standard for full-field clinical electroretinography (2008 update). Doc. Ophthalmol. 118, 69-77 (2009).

20. Vincent, A. et al. A phenotype-genotype correlation study of X-linked retinoschisis. Ophthalmology 120, 1454-1464 (2013).

21. Bowles, K. et al. X-linked retinoschisis: RS1 mutation severity and age affect the ERG phenotype in a cohort of 68 affected male subjects. Invest. Ophthalmol. Vis. Sci. 52, 9250-9256 (2011).

22. Hafler, B. P. Clinical progress in inherited retinal degenerations: gene therapy clinical trials and advances in genetic sequencing. Retina $37,417-423$ (2017). 